$16^{\text {th }}$ International Congress of Metrology, 04001 (2013)

DOI: $10.1051 /$ metrology/201304001

(C) Owned by the authors, published by EDP Sciences, 2013

\title{
Uncertainty evaluation by means of joint possibility distributions
}

\author{
Alessandro Ferrero ${ }^{1, a}$, Marco Prioli ${ }^{1}$, and Simona Salicone ${ }^{1}$ \\ ${ }^{1}$ Politecnico di Milano, Dept. of Electronics, Information and Bioengineering, Piazza Leonardo da Vinci 32, 20133 Milano, Italy
}

\begin{abstract}
The recent theories of evidence and possibility represent an interesting generalization of the wellknow probability theory. Therefore, they appear to be a promising mathematical framework for the expression of uncertainty in measurement, capable of overcoming the limitations of the presently followed probability approach when uncompensated systematic effects are present. This paper proposes an example of uncertainty evaluation framed within these new theories and an experimental validation that shows the correctness and effectiveness of the proposed approach.
\end{abstract}

\section{Introduction}

In the recent years, an interesting discussion has started about the mathematical framework suggested by the Guide to the Expression of Uncertainty in Measurement (GUM) [1] to evaluate and express uncertainty in measurement. While the uncertainty concept has been universally accepted, the purely probabilistic approach suggested by the GUM has been considered too restrictive, since it implies the identification and compensation of all significant systematic effects. Especially in industrial applications, this may not be cost effective, and therefore a more general mathematical framework should be considered to evaluate the contributions of uncompensated systematic effects and their combination with the random ones on measurement uncertainty [2].

The theory of evidence [3], which encompasses probability as a particular case, and the theory of possibility (that is another particular case of evidence) have been considered by several researchers [4-7] as a promising mathematical tool to overcome the limitations of the GUM probabilistic approach. Several interesting applications are already available [8-12], thus proving the interest toward this more general approach and its capability of providing an effective representation of measurement results together with their uncertainty.

This paper, after having briefly recalled the fundamentals of the evidence approach in terms of Random-Fuzzy Variables (RFVs) [6, 13], proposes a simple example of application, aimed at showing the effectiveness of this approach in a practical case.

\section{RFV approach}

This new approach to measurement uncertainty is built around the concept of Random-Fuzzy Variable. RFVs are

\footnotetext{
${ }^{\mathrm{a} C}$ Corresponding author: alessandro.ferrero@polimi.it
}

type-2 fuzzy variables [14], defined to represent a measurement result $[6,13]$ (i.e. the measured value together with its uncertainty). The very basic concept of RFVs is briefly recalled here, without entering too many mathematical details, for which the reader is addressed to the available literature.

Fig. 1 shows an RFV and the possibility distributions (PDs) that define the RFV itself: the "internal PD" $r_{\text {int }}$, representing the non-random contributions to uncertainty, and the "external PD" $r_{\text {ext }}$, representing all contributions to uncertainty $[6,13]$. This last PD is provided by the combination of $r_{\text {int }}$ with the "random PD" $r_{\text {ran }}$ that represents only the random contributions to uncertainty $[6,13]$.

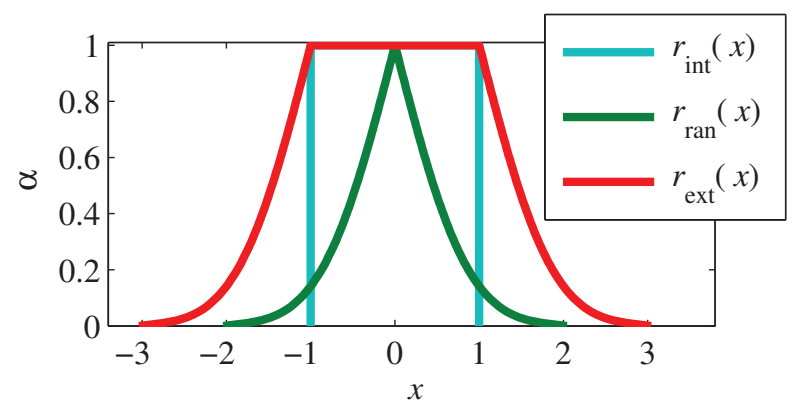

Figure 1. Example of RFV (red + cyan lines) and its PDs: $r_{\text {int }}(x)$ (cyan line), $r_{\text {ran }}(x)$ (green line), $r_{\text {ext }}(x)$ (red line).

Starting from the available metrological information, both the internal and random PDs can be built $[6,15]$. In particular, while the internal PD is generally built directly in terms of a possibility distribution, the random PD is often induced by a probability distribution function (pdf). This can be obtained by applying suitable probabilitypossibility transformations to the pdf representing the random phenomena $[16,17]$.

It can be proved [2] that the $\alpha$-cut of a PD, i.e. its cut at level $\alpha$, extends to the possibility theory the concept of 


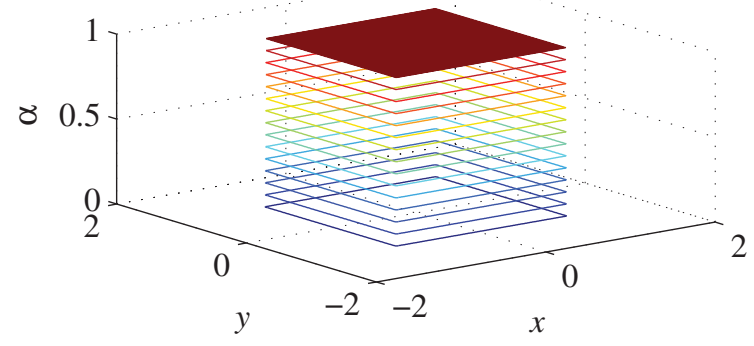

Figure 2. $r_{X, Y}$ induced by $T_{\min }$ when the PDs $r_{X}$ and $r_{Y}$ are independent and both given by $r_{\text {int }}$ of Fig. 1 .

confidence interval. Therefore, for the sake of simplicity, it is possible to state that the $\alpha$-cut of the external PD represents the interval with confidence $1-\alpha$ associated with the considered measurement result $[2,13]$.

A specific mathematical background has been developed concerning the RFVs combination [13, 18, 19]. A general rule to perform RFVs combination is to apply, to the PDs (internal or random) that compose the RFVs, the Zadeh's extension principle [20]:

$$
r_{Z}(z)=\sup _{z=f(x, y)}\left(r_{X, Y}(x, y)\right),
$$

where $f$ is the measurement function through which the RFVs have to be combined, and $r_{X, Y}$ is the joint possibility distribution of the considered variables $X$ and $Y$. Therefore, before applying the Zadeh's extension principle, the joint PD $r_{X, Y}$ has to be evaluated. In the possibility framework, this can be done by applying suitable mathematical operators $T$, called $t$-norms [21], to the uni-variate PDs $r_{X}$ and $r_{Y \mid X=x}$ :

$$
r_{X, Y}(x, y)=T\left(r_{X}(x), r_{Y \mid X=x}(y)\right)
$$

Considering $r_{Y \mid X=x}$, instead of simply $r_{Y}$, is a more general formulation that takes into account the possible dependence of $Y$ on $X[18,19]$.

Recently, it has been shown that the choice of the specific $t$-norm $T$ to be employed in (2) is a critical point in RFVs combination $[18,19]$. In fact, a different $t$ norm has to be applied to the internal and random PDs to preserve the non-random and random nature of the uncertainty contributions represented by $r_{\text {int }}$ and $r_{\text {ran }}$, respectively. Without entering the mathematical details, the $t$-norm $\min \left(T_{\min }(a, b)=\min (a, b)\right)$ [21] combines effectively the non-random uncertainty contributions [18]. Fig. 2 shows the joint PD $r_{X, Y}$ induced by $T_{\min }$ when the PDs $r_{X}$ and $r_{Y \mid X=x}=r_{Y}$ are independent and both given by the uniform PD $r_{\text {int }}$ of Fig. 1. It can be noted that the obtained $r_{X, Y}$ is the least specific joint PD (i.e. the largest one), being $T_{\min }$ the largest possible $t$-norm.

When the random contributions to uncertainty are considered, a smaller $t$-norm than $T_{\min }$ has to be considered. In fact [19], an effective combination of such contributions can be obtained choosing $T$ among the Frank parametric

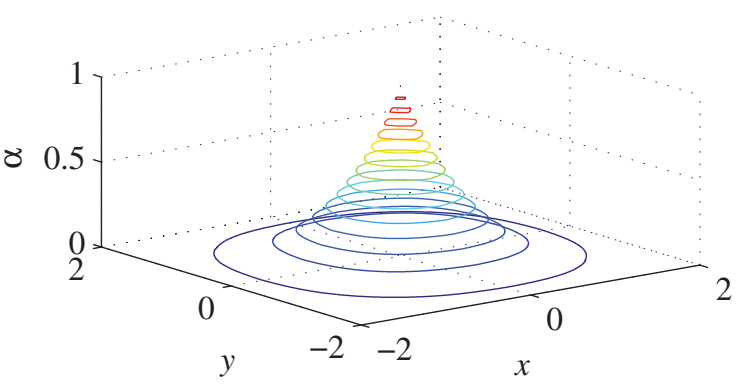

Figure 3. $r_{X, Y}$ induced by $T_{\gamma=0.05}^{F}$ and when the PDs $r_{X}$ and $r_{Y}$ are independent and both given by $r_{\text {ran }}$ of Fig. 1 .

family of $t$-norms, defined as [21, 22]:

$$
T_{\gamma}^{F}(a, b)= \begin{cases}T_{\min }(a, b)=\min (a, b) & \text { if } \gamma=0 \\ T_{\text {prod }}(a, b)=a \cdot b & \text { if } \gamma=1 \\ T_{L}(a, b)=\max (0, a+b-1) & \text { if } \gamma=\infty \\ \log _{\gamma}\left(1+\frac{\left(\gamma^{a}-1\right) \cdot\left(\gamma^{b}-1\right)}{\gamma-1}\right) & \text { otherwise }\end{cases}
$$

Fig. 3 shows the joint PD $r_{X, Y}$ induced by $T_{\gamma=0.05}^{F}$ and when the PDs $r_{X}$ and $r_{Y \mid X=x}=r_{Y}$ are independent and both given by the PD $r_{\text {ran }}$ of Fig. 1 .

The mathematical background developed in the literature and recalled above provides the theoretical framework into which measurement uncertainty can be evaluated and expressed, also in the presence of uncompensated systematic contributions.

\section{Considered example}

In order to show the effectiveness of the RFV approach in the representation and evaluation of measurement uncertainty, a simple, though meaningful, metrological example has been considered. In this Section, the considered experimental setup and the uncertainty evaluation procedure are firstly discussed. The obtained results are then shown and discussed.

\subsection{Experimental setup}

The considered metrological example consists in the characterization of a resistive voltage divider, whose schematic is shown in Fig. 4. Two configurations of the voltage

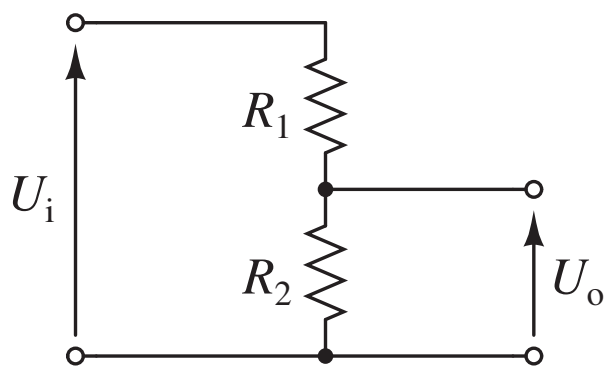

Figure 4. Schematic of the voltage divider. 


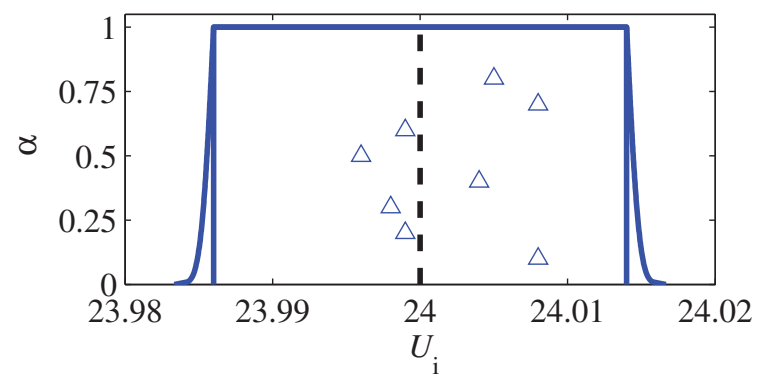

Figure 5. RFV associated with $U_{\mathrm{i}}$ (continuous lines), $\hat{U}_{\mathrm{i}}$ (dashed line), and $U_{\mathrm{i}}$ experimental values (triangles) for configuration $A$.

divider have been considered: configuration $A$ defined by $R_{1}=R_{2}=10 \mathrm{k} \Omega$ and configuration $B$ defined by $R_{1}=10 \mathrm{k} \Omega$ and $R_{2}=1 \mathrm{k} \Omega$.

In order to obtain the reference value of the voltage ratio $k=U_{\mathrm{o}} / U_{\mathrm{i}}$, the divider has been supplied by the DC voltage $U_{\mathrm{i}}$ generated by a Fluke $5500 \mathrm{~A}$ calibrator and the output voltage $U_{\mathrm{o}}$ has been measured by means of a Fluke $8508 \mathrm{~A}$ reference multimeter. The reference value $\hat{k}$ of the divider is obtained as $\hat{k}=\hat{U}_{\mathrm{o}} / \hat{U}_{\mathrm{i}}$, where $\hat{U}_{\mathrm{i}}$ is the input voltage set on the calibrator and $\hat{U}_{\mathrm{o}}$ is the measured output voltage. Since the Fluke calibrator and multimeter represent the benchmark instrumentation, $\hat{U}_{\mathrm{i}}, \hat{U}_{\mathrm{o}}$ and $\hat{k}$ can be considered as reference values, not affected by uncertainty.

The input and output voltages have been measured also by means of 8 Leader 856 digital multimeters, thus obtaining 8 measured values $U_{\mathrm{i}}$ and 8 measured values $U_{\mathrm{o}}$ which provide $8^{2}=64$ values of $k$. Due to measurement uncertainty, the values $U_{\mathrm{i}}, U_{\mathrm{o}}$ and $k$ measured by means of the Leader multimeters provide only an estimate of the quantities $\hat{U}_{\mathrm{i}}, \hat{U}_{\mathrm{o}}$ and $\hat{k}$. The main contributions to uncertainty due to the Leader multimeters are a systematic offset contribution and a random contribution due to the intrinsic variability of repeated measurements of the same quantity. According to the manufacturer's specifications, the systematic contribution introduced by a multimeter is supposed to lie in the interval given by $\pm 0.05 \%$ of the reading \pm 2 digits. The measurements variability has been experimentally evaluated and found to lie in the interval \pm 2 digits.

Moreover, when the same multimeter is employed to measure both $U_{\mathrm{i}}$ and $U_{\mathrm{o}}$, the systematic effects affecting the two measured values are correlated, and this correlation has to be estimated and taken into account in uncertainty evaluation.

\subsection{Uncertainty evaluation}

In order to quantify the uncertainty contributions affecting $k$, the uncertainty contributions affecting $U_{\mathrm{i}}$ and $U_{\mathrm{o}}$ have to be propagated considering the measurement function $k=U_{\mathrm{o}} / U_{\mathrm{i}}$. According to the considerations made in Sec. 2, this can be done in the RFV framework. Therefore, the assumptions about the possible range of values of the systematic and random contributions affecting $U_{\mathrm{i}}$ and $U_{\mathrm{o}}$ must be represented by means of RFVs.

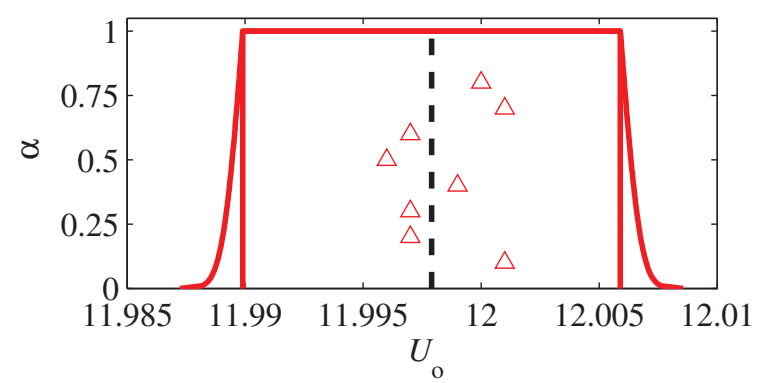

Figure 6. RFV associated with $U_{\mathrm{o}}$ (continuous lines), $\hat{U}_{\mathrm{o}}$ (dashed line), and $U_{\mathrm{o}}$ experimental values (triangles) for configuration $A$.

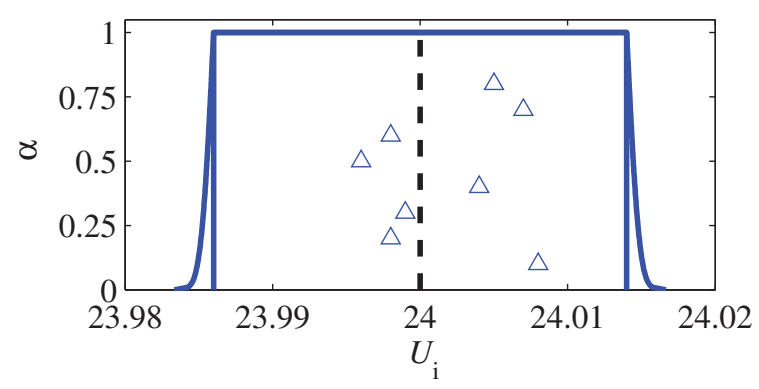

Figure 7. RFV associated with $U_{\mathrm{i}}$ (continuous lines), $\hat{U}_{\mathrm{i}}$ (dashed line), and $U_{\mathrm{i}}$ experimental values (triangles) for configuration $B$.

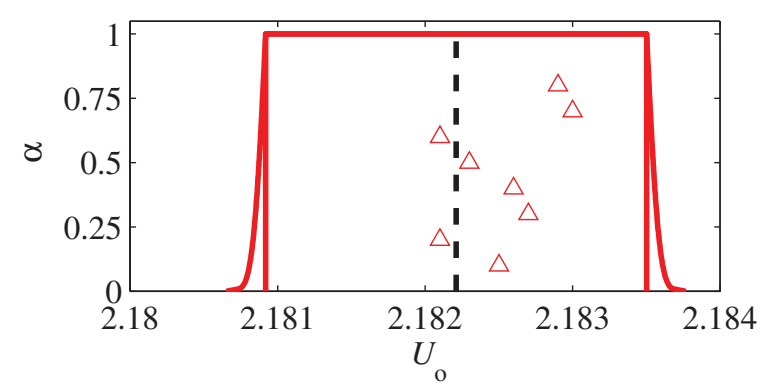

Figure 8. RFV associated with $U_{\mathrm{o}}$ (continuous lines), $\hat{U}_{\mathrm{o}}$ (dashed line), and $U_{\mathrm{o}}$ experimental values (triangles) for configuration $B$.

When the systematic contribution is considered and only the manufacturer's specifications are available, the only relevant available information is the interval within which the contribution itself is supposed to lie. No information is available to assign any specific probability distribution over this interval, so that, in the framework of the theory of evidence, its distribution over this interval can be represented by a non-informative (rectangular) possibility distribution $[13,15]$, that is the upper envelope of all possible families of probability distributions over the given interval [16]. For this reason, the internal PDs of $U_{\mathrm{i}}$ and $U_{\mathrm{o}}$ are assumed to be rectangular PDs whose width is given by $\pm 0.05 \%$ of the reading \pm 2 digits, according to the manufacturer's specifications.

When the random contribution is considered, according to the experimental evaluation of measurements variability, it can be assumed to take values within a known 


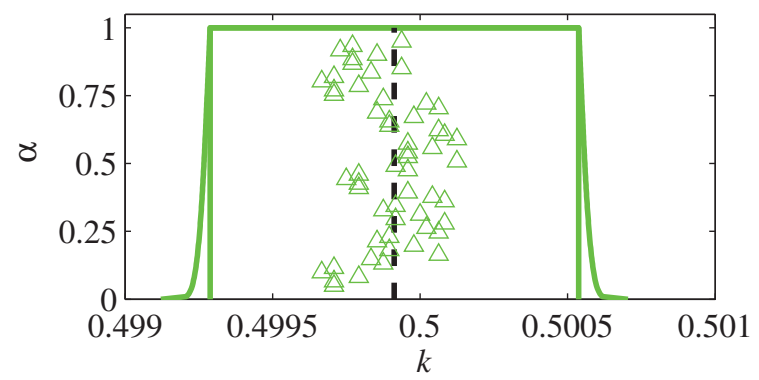

Figure 9. RFV associated with $k$ (continuous lines), $\hat{k}$ (dashed line), and $k$ experimental values (triangles) for configuration $A$ and using different multimeters to measure $U_{\mathrm{i}}$ and $U_{\mathrm{o}}$.

interval and distribute normally over that interval. Therefore, the random PDs of $U_{\mathrm{i}}$ and $U_{\mathrm{o}}$ are assumed to be induced by normal probability distributions [13, 16, 17] whose $99 \%$ confidence interval $\pm 3 \sigma$ is given by the experimental interval \pm 2 digits.

The composition of the internal PDs of $U_{\mathrm{i}}$ and $U_{\mathrm{o}}$ with their random PDs provide the final RFVs of $U_{i}$ and $U_{o}$ [13]. In general, the mean value of the obtained RFV is centered on the measured value and the unknown value of the measurand is expected to lie within the $\alpha$-cuts of the obtained RFV. This procedure would lead to an RFV for each measured value of $U_{\mathrm{i}}$ and $U_{\mathrm{o}}$. However, in this particular case, the measurand value is known, since it has been generated or measured by reference instruments. Therefore, for the sake of simplicity, the obtained RFVs have been centered on the reference values $\hat{U}_{\mathrm{i}}$ and $\hat{U}_{\mathrm{o}}$. Thus, a single RFV can be considered for $U_{\mathrm{i}}$ and $U_{\mathrm{o}}$ respectively, and all measured values are expected to lie within the $\alpha$ cuts of these RFVs. This simpler procedure has also the advantage that the interpretation of the obtained results is more immediate, as shown in the following figures.

Figures 5 and 6 show the RFVs associated with $U_{\mathrm{i}}$ and $U_{\mathrm{o}}$ for configuration $A$ of the voltage divider, while Figs. 7 and 8 show the same RFVs for configuration $B$. The same figures show also the experimental values of $U_{\mathrm{i}}$ and $U_{\mathrm{o}}$ provided by the 8 multimeters ${ }^{1}$.

It can be seen that some multimeters provide values that are lower than the reference ones while other multimeters provide values that are greater than the reference ones but all values are well inside the RFVs of $U_{\mathrm{i}}$ and $U_{\mathrm{o}}$. The RFVs show also that the random contribution has a limited impact on uncertainty with respect to the systematic contribution.

\subsection{Results}

According to the consideration made in Sec. 2, the RFVs of $U_{\mathrm{i}}$ and $U_{\mathrm{o}}$ can be combined through the measurement function $k=U_{\mathrm{o}} / U_{\mathrm{i}}$ to provide the RFV of $k$. The possible correlation of $U_{\mathrm{i}}$ and $U_{\mathrm{o}}$ RFVs must be considered in this combination. The random PDs of $U_{\mathrm{i}}$ and $U_{\mathrm{o}}$ are, of

\footnotetext{
${ }^{1}$ In all figures, the experimental values have been plotted in different vertical positions only to clearly distinguish them.
}

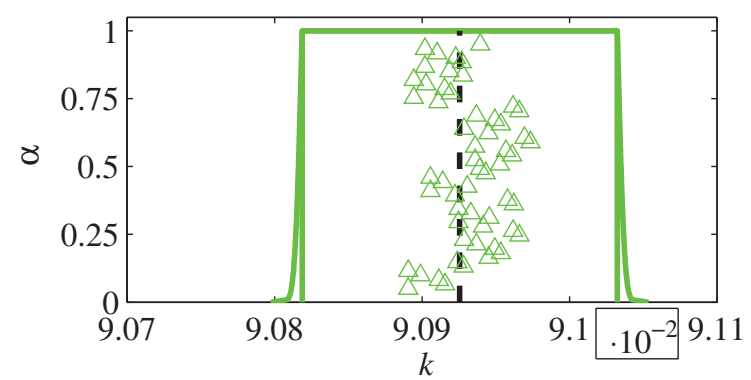

Figure 10. RFV associated with $k$ (continuous lines), $\hat{k}$ (dashed line), and $k$ experimental values (triangles) for configuration $B$ and using different multimeters to measure $U_{\mathrm{i}}$ and $U_{\mathrm{o}}$.

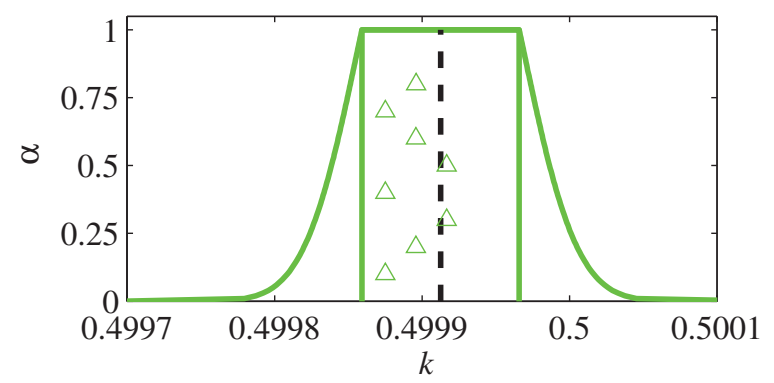

Figure 11. RFV associated with $k$ (continuous lines), $\hat{k}$ (dashed line), and $k$ experimental values (triangles) for configuration $A$ and using the same multimeters to measure $U_{\mathrm{i}}$ and $U_{\mathrm{o}}$.

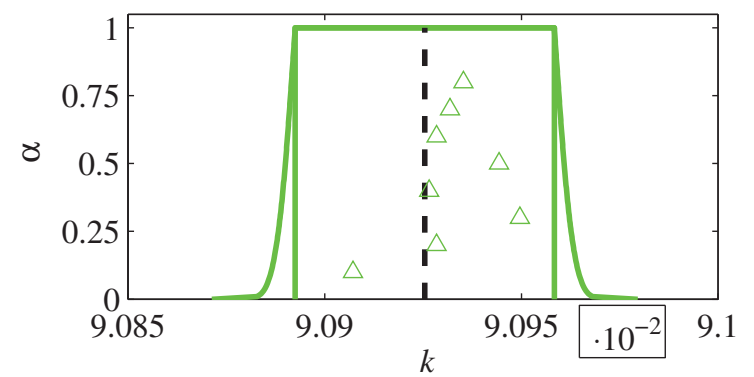

Figure 12. RFV associated with $k$ (continuous lines), $\hat{k}$ (dashed line), and $k$ experimental values (triangles) for configuration $B$ and using the same multimeters to measure $U_{\mathrm{i}}$ and $U_{\mathrm{o}}$.

course, not correlated due to the independent random contributions which originate them. On the contrary, the internal PDs of $U_{\mathrm{i}}$ and $U_{\mathrm{o}}$ may show some correlation. In fact, the internal PDs are correlated when $U_{\mathrm{i}}$ and $U_{\mathrm{o}}$ are measured by the same digital multimeter since, in this case, the systematic contributions affecting $U_{\mathrm{i}}$ and $U_{\mathrm{o}}$ assume very similar values. The correlation between the internal PDs of $U_{\mathrm{i}}$ and $U_{\mathrm{o}}$ has been experimentally evaluated and found to be $\rho_{A}=0.98$ for configuration $A$ of the voltage divider and $\rho_{B}=0.7$ for configuration $B$. These values are not surprising since, for configuration $A, U_{\mathrm{i}}$ and $U_{\mathrm{o}}$ are measured with the same voltage range setting and, therefore, the multimeter introduce almost the same systematic contributions. For configuration $B, U_{\mathrm{i}}$ and $U_{\mathrm{o}}$ are measured with different voltage range settings. Therefore, since the 
multimeter changes its configuration to measure $U_{\mathrm{i}}$ and $U_{\mathrm{o}}$, the correlation between the measurement results is not as strong as in the previous case.

According to the mathematics recalled in Sec. 2, the joint possibility distribution $r_{U_{\mathrm{i}}, U_{\mathrm{o}}}$ has been obtained under the different considered correlation assumptions. Then (1) has been computed, with $f\left(U_{\mathrm{i}}, U_{\mathrm{o}}\right)=U_{\mathrm{o}} / U_{\mathrm{i}}$.

Figures 9 and 10 show the RFVs associated with $k$ for configuration $A$ and $B$, respectively, when different multimeters are used to measure $U_{\mathrm{i}}$ and $U_{\mathrm{o}}$. Of course, the mean values of these RFV coincides with the reference values $\hat{k}$. The same figures show also the experimental values of $k$ for the respective configurations of the voltage divider.

Once again, it can be seen that all the experimental $k$ values are well inside the RFV of $k$ for both configurations $A$ and $B$. Therefore, the RFVs of $U_{\mathrm{i}}$ and $U_{\mathrm{o}}$ have been correctly combined through the measurement function $k=U_{\mathrm{o}} / U_{\mathrm{i}}$. Also in this case, the random contribution has a limited impact on uncertainty with respect to the systematic contribution.

Figures 11 and 12 show the RFVs associated with $k$ for configuration $A$ and $B$, respectively, when the same multimeters are used to measure $U_{\mathrm{i}}$ and $U_{\mathrm{o}}$. Of course, the mean values of these RFV coincides with the reference values $\hat{k}$. The same figures show also the experimental values of $k$ for the respective configurations of the voltage divider.

Comparing Fig. 11 with Fig. 9 and Fig. 12 with Fig. 10, it can be seen that the correlation between the internal PDs of $U_{\mathrm{i}}$ and $U_{\mathrm{o}}$ has significantly reduced the width of the internal PD of $k$, as expected. Anyway, all experimental $k$ values are correctly inside the RFVs of $k$. In this case, since the impact of the systematic contribution to uncertainty is lower, the impact of the random contribution appears to be more significant. For all these reasons, it can be stated that the RFVs of $U_{\mathrm{i}}$ and $U_{\mathrm{o}}$ have been correctly combined through the measurement function $k=U_{\mathrm{o}} / U_{\mathrm{i}}$ also in presence of correlated systematic contributions to uncertainty.

\subsection{Discussion}

The results reported in the previous sections prove that RFVs provide a correct and effective representation of the measurement results together with the related uncertainty. It is here worth discussing an additional point related to the different way systematic effects are considered in a purely probabilistic approach and in the employed RFV approach.

When a whole population of multimeters is considered, the offset systematic effects distribute over the interval provided by the manufacturer with a probability distribution that is usually considered uniform when no additional information is available. This is clearly shown by Figs. 5, 6, 7, 8, that confirm also, in the limited number of considered multimeters, the uniform probability assumption.

This leads often to model the uncompensated systematic effect with a uniform probability distribution over the given interval. This is acceptable as far as this assumption is valid, and if one wants to represent the contribution to uncertainty of the offset error of the whole population of multimeters. Picking a single multimeter corresponds to extracting a sample from this distribution. Therefore, if one wants to evaluate the uncertainty of a single measured value, this approach is equivalent to the one in terms of RFVs, as proved by the values plotted in all considered figures.

On the other hand, if only one multimeter is employed and no assumption can be made about the way the offset error distributes in the interval provided by the manufacturer, if the measured value is combined with other measured values and one wants to evaluate how the unknown offset error affects the final measurement uncertainty, the systematic nature of this effect has to be taken into account. The offset error will take a unique, though unknown value from the considered population of offset errors, and this value will not change during the measurement process. Therefore, its contribution to uncertainty can be represented only by a uniform possibility distribution, since the only available information is that it belongs to the interval provided by the manufacturer.

This is well proved by the RFVs shown in the previous sections. If the same two multimeters are employed to measure the voltage ratio $k$, they will always provide the same estimate, that is the same triangle inside the given RFV. A probability distribution can be assumed if and only if every time a new measurement is performed, a new pair of multimeters is randomly selected by the available population. Since this is generally not the case, the representation of uncertainty in terms of RFVs (that is framed within the theory of possibility) appears to be more effective than the one suggested by the GUM.

\section{Conclusions}

The evaluation of measurement uncertainty in terms of RFVs has been considered in this paper, as an interesting generalization of the purely probabilistic GUM approach when significant systematic effects are not compensated for.

The theoretical approach has been applied to a very simple, though significant practical example, where the offset error of the employed digital multimeters has not been compensated, but only estimated starting from the manufacturer specifications.

The experimental results show the practical utility of the proposed method, which appears to be more effective than the method proposed by the GUM when a single measurement performed by a single pair of instruments is considered.

\section{References}

[1] JCGM 100:2008, Evaluation of Measurement DataGuide to the Expression of Uncertainty in Measurement, (GUM, originally published in 1993), Joint Committee for Guides in Metrology (2008)

[2] A. Ferrero, S. Salicone, IEEE Trans. Instrum. Meas. 61, 2167 (2012) 
[3] G. Shafer, A Mathematical Theory of Evidence (Princeton Univ. Press, Princeton, NJ, USA, 1976)

[4] G. Mauris, L. Berrah, L. Foulloy, A. Haurat, IEEE Trans. Instrum. Meas. 49, 89 (2000)

[5] M. Urbanski, J. Wasowsky, Measurement, Elsevier Science 34, 67 (2003)

[6] A. Ferrero, S. Salicone, IEEE Trans. Instrum. Meas. 53, 1370 (2004)

[7] A. Ferrero, S. Salicone, The theory of evidence for the expression of uncertainty in measurement, in 12 International Metrology Congress (Lyon, France, 2005), pp. $1-6$

[8] A. Ferrero, S. Salicone, An innovative method for the comparison of measurement results, in 13 International Congress of Metrology (Lille, France, 2007), pp. 1-5

[9] A. Ferrero, M. Prioli, S. Salicone, IEEE Trans. Instrum. Meas. 61, 2972 (2012)

[10] Q. Zhu, Z. Jiang, Z. Zhao, H. Wang, Review of Scientific Instruments 77 (2006)

[11] M. Pertile, M. De Cecco, L. Baglivo, IEEE Trans. Instrum. Meas. 59, 2816 (2010)

[12] A. Ferrero, R. Ferrero, M. Prioli, S. Salicone, An extension of the conditional probability concept to Random-Fuzzy Variables representing measurement result, in 15 International Congress of Metrology (Paris, France, 2011), pp. 1-6

[13] S. Salicone, Measurement Uncertainty: an approach via the mathematical theory of evidence, Springer series in reliability engineering (Springer, New York, NY, USA, 2007), ISBN 0387306552

[14] G.J. Klir, B. Yuan, Fuzzy sets and fuzzy logic. Theory and applications (Prentice Hall PTR, Englewood Cliffs, NJ, USA, 1995), ISBN 978-0-13-101171-7

[15] A. Ferrero, S. Salicone, IEEE Trans. Instrum. Meas. 58, 365 (2009)

[16] D. Dubois, L. Foulloy, G. Mauris, H. Prade, Reliable Computing. Kluwer Academic Publishers 10, 273 (2004)

[17] A. Ferrero, M. Prioli, S. Salicone, B. Vantaggi, IEEE Trans. Instrum. Meas. 62, 982 (2013)

[18] A. Ferrero, M. Prioli, S. Salicone, IEEE Trans. Instrum. Meas. 4, 720 (2013)

[19] A. Ferrero, M. Prioli, S. Salicone (2013), accepted for publication on IEEE Trans. Instrum. Meas.

[20] L.A. Zadeh, Fuzzy Sets and Systems 1, 3 (1978)

[21] E.P. Klement, R. Mesiar, E. Pap, Triangular Norms (Kluwer, Dordrecht, Netherlands, 2000), ISBN 0792364163

[22] M. Frank, Aequationes mathematicae 19, 141 (1979) 